\title{
Ectomesenchymal Chondromyxoid Tumor
}

National Cancer Institute

\section{Source}

National Cancer Institute. Ectomesenchymal Chondromyxoid Tumor. NCI Thesaurus.

Code $C 121785$.

A rare, well circumscribed, non-encapsulated tumor that arises in the oral cavity, most often the anterior tongue. It is characterized by the presence of round to spindle cells in a chondromyxoid or hyalinized stroma. Recurrences have been observed in a minority of patients. 